\section{(6) OPEN ACCESS}

\title{
Synovial tissue volume: a treatment target in knee osteoarthritis (OA)
}

\author{
Terence W O'Neill, 1,2,3 Matthew J Parkes, ${ }^{1,2}$ Nasimah Maricar, ${ }^{1,2}$ \\ Elizabeth J Marjanovic, ${ }_{1}^{1,2}$ Richard Hodgson, ${ }^{4}$ Andrew D Gait, ${ }^{4}$ Timothy F Cootes, ${ }^{4}$ \\ Charles E Hutchinson, ${ }^{5}$ David T Felson ${ }^{1,2,6}$
}

\begin{abstract}
Handling editor Tore K Kvien
For numbered affiliations see end of article.

Correspondence to Professor Terence O'Neill, Faculty of Medical and Human Sciences, Arthritis Research UK Centre for Epidemiology, Institute of Inflammation and Repair, Manchester Academic Health Science Centre, The University of Manchester, Manchester, M13 9PT, UK; terence.o'neill@manchester. ac.uk
\end{abstract}

Received 3 November 2014 Revised 22 April 2015 Accepted 23 April 2015 Published Online First 26 June 2015
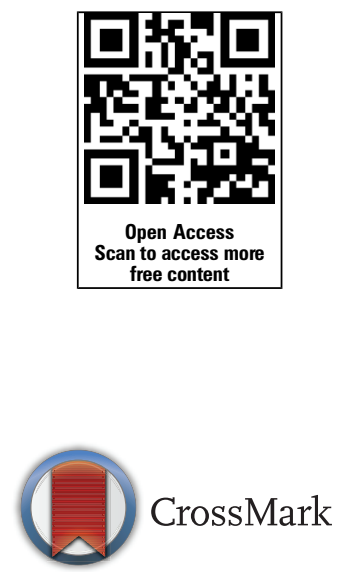

\footnotetext{
To cite: O'Neill TW,

Parkes MJ, Maricar N, et al. Ann Rheum Dis

2016:75:84-90.
}

\section{ABSTRACT}

Background Synovitis occurring frequently in osteoarthritis (OA) may be a targeted outcome. There are no data examining whether synovitis changes following intra-articular intervention.

Methods Persons aged 40 years and older with painful knee OA participated in an open label trial of intraarticular steroid therapy. At all time points they completed the Knee Injury and Osteoarthritis Outcome Score (KOOS) questionnaire. They had a contrastenhanced (CE) MRI immediately prior to an intraarticular steroid injection with a repeat scan within 20 days. Response status was assessed using the Osteoarthritis Research Society International (OARSI) response criteria. OARSI responders were followed until their pain relapsed either within $20 \%$ of baseline or 6 months, shortly after which a third MRI was performed. Synovial tissue volume (STV) was measured on postcontrast knee images. We looked at changes in the STV and in pain, and their association.

Results 120 subjects with preinjection and postinjection CE MRI were followed. Their mean age was 62.3 years $(S D=10.3)$ and $62(52 \%)$ were women. The median time between injection and follow-up scan was 8 days (IQR 7-14 days). 85/120 (71\%) were OARSI responders. Pain decreased (mean change in KOOS= +23.9; $95 \% \mathrm{Cl} 20.1$ to 27.8, $\mathrm{p}<0.001$ ) following steroid injection, as did mean STV (mean change= $-1071 \mathrm{~mm}^{3} ; 95 \% \mathrm{Cl}-1839 \mathrm{~mm}^{3}$ to $-303 \mathrm{~mm}^{3}$, $\mathrm{p}=0.01$ ). Of the 80 who returned for a third MRI, pain relapsed in 57, and in the 48 of those with MRI data, STV increased between follow-up and final visit $\left(+1220 \mathrm{~mm}^{3} ; 95 \% \mathrm{Cl} 25 \mathrm{~mm}^{3}\right.$ to $\left.2414 \mathrm{~mm}^{3}, \mathrm{p}=0.05\right)$. 23 were persistent responders at 6 months and, in these, STV did not increase (mean change $=-202 \mathrm{~mm}^{3}$; $95 \% \mathrm{Cl}-2008 \mathrm{~mm}^{3}$ to $1604 \mathrm{~mm}^{3}, \mathrm{p}=0.83$ ).

Controlling for variation over time, there was a significant association between synovitis volume and KOOS pain (b coefficient-change in KOOS pain score per $1000 \mathrm{~mm}^{3}$ change in STV $=-1.13 ; 95 \% \mathrm{Cl}-1.87$ to -0.39, $p=0.003)$, although STV accounted for only a small proportion of the variance in change in pain.

Conclusions Synovial tissue volume in knee OA shrinks following steroid therapy, and rebounds in those whose pain relapses. It can be considered a treatment target in symptomatic knee OA.

Trial registration number ISRCTN07329370.

\section{INTRODUCTION}

Synovitis is a well-recognised feature of knee osteoarthritis (OA). On arthroscopy, synovitis is seen in approximately $50 \%$ of the knees of patients with painful $\mathrm{OA}$ and in an even higher percentage using MRI. ${ }^{1}{ }^{2}$ Loeuille et $a l^{3}$ comparing MRI, histologic and arthroscopic appearance of synovium in persons with symptomatic knee OA reported a high correlation between the degree of synovial thickening on MRI and macroscopic scoring of synovitis by an arthroscopist $(r=0.58)$. Thickness was also correlated with infiltration of inflammatory cells into the subsurface layers of synovium $(r=0.46)$. Thus, synovial thickening observed from gadolinium-enhanced MRI correlates with macroscopic and microscopic evidence of synovitis and is typically referred to as synovitis.

Using non-contrast enhanced MRI, Hill and colleagues reported that synovitis was correlated crosssectionally with the severity of knee pain in persons with knee OA. ${ }^{4}$ Hill also found a modest correlation $(\mathrm{r}=0.21)$ between change in synovitis on MRI with change in severity of knee pain over time in 270 persons with symptomatic knee OA who had undergone serial MRIs. ${ }^{5}$ These findings have been corroborated by Zhang and colleagues using data from serial MRIs in the Multicenter Osteoarthritis Study (MOST). ${ }^{6}$ However, the results from these studies are limited by the use of non-contrast enhanced MRI to characterise synovitis. Using a non-contrast technique, it is difficult to distinguish synovitis from effusion, and some areas of synovitis are impossible to differentiate from surrounding structures. ${ }^{2}{ }^{7}{ }^{7}{ }^{8}$ Contrast enhances the appearance of synovium without showing the other surrounding structures, and its use is critical to best identify synovitis. Baker et al ${ }^{9}$ reported on a subset of the MOST cohort who had gadolinium-enhanced MRIs and found that the contrast-enhanced synovitis appeared to be strongly associated with pain. Synovitis was far more prevalent in the knee pain group.

Intra-articular corticosteroids have long been a mainstay of OA treatment, thought to act through their anti-inflammatory effect. In a meta-analysis of randomised trials comparing corticosteroid with placebo injections for the treatment of knee OA, Arroll and Goodyear-Smith ${ }^{10}$ reported the superiority of corticosteroid injections. The treatment effect was large, and the number needed to treat ranged from 1.3 to 3.5 . In meta-analyses, steroids are more effective than placebo injection for up to 2 weeks after steroid injection, but, for many patients, the beneficial effect is much longer. ${ }^{10}$ In rheumatoid arthritis, a reduction in synovial 
volume has been seen after intra-articular steroid injections, but the effect of treatment on synovitis in OA has not, to our knowledge, been studied. ${ }^{11}$

Given the interest in testing OA treatments targeting synovial inflammation, knowledge of whether measurable reduction in synovitis occurs with appropriate treatment is needed. Such data are important to serve as evidence that synovial volume changes in response to intervention, and would be a key milestone in considering synovial volume as an outcome measure in clinical trials of knee OA. Also, if synovitis diminishes, it is unclear whether this is correlated with pain reduction. Further, there is no evidence of longer term effects of intra-articular steroids on structure and particularly whether synovitis rebounds after treatment.

The aims of this study were to determine (i) whether synovial tissue volume (STV) as assessed using contrast-enhanced MRI changes in response to intra-articular steroid therapy and (ii) whether change in symptoms of pain correlates with changes in STV. Our study design, in which we examined MRIs before and after steroid injection and also obtained a third MRI on relapse of pain, allowed us also to examine the relation of pain fluctuation to change in STV.

\section{METHODS \\ Subjects}

Men and women aged 40 years and over were recruited from both primary and secondary care clinics for participation in an open label study to observe the efficacy of intra-articular steroid therapy in symptomatic knee OA (ISRCTN: 07329370). Subjects were included if they reported moderate knee pain for more than $48 \mathrm{~h}$ in the previous 2 weeks or scored greater than 7 out of 32 on the Knee Injury and Osteoarthritis Outcome Score (KOOS) questionnaire, questions P2-P9 (Question P1 relates to frequency of knee pain, which is irrelevant given the inclusion criteria on pain frequency). Inclusion criteria included imaging confirmation of $\mathrm{OA}$ either radiologically (in any joint on anterio-posterior (AP), skyline or lateral knee radiographs obtained within the previous 2 years) with a Kellgren-Lawrence score of two or more or, on MRI scan or at arthroscopy. For MRI and arthroscopy, we required typical changes of OA with at least cartilage loss present. Exclusion criteria included the presence of secondary OA from gout, previous septic arthritis or inflammatory arthritis, injection with hyaluronic acid or steroid injection within the previous 3 months, history of knee surgery within the previous 6 months, concurrent lifethreatening illness and any contraindication to MRI scanning. Subjects were provided with an information sheet about the study and those who agreed to take part subsequently provided written informed consent.

\section{Screening and baseline assessment}

Those who were interested initially attended a screening visit to determine eligibility. Where subjects had not had a knee radiograph in the previous 2 years, or other imaging evidence of OA, knee radiographs were performed. Blood was also taken to assess renal function. Those with an estimated glomerular filtration rate less than $40 \mathrm{~mL} / \mathrm{min}$ were subsequently excluded from the study. Those who were eligible and fulfilled the inclusion/exclusion criteria were invited to attend a baseline visit. Subjects also completed a series of questionnaires including the KOOS pain scale and a visual analogue scale (VAS) score for pain during an activity that a patient nominated as being most troublesome $\left(\mathrm{VAS}_{\mathrm{NA}}\right){ }^{12}$ Subjects had a CE MRI scan performed with gadolinium as the contrast agent followed by an intra-articular steroid injection within a couple of hours (see below).

\section{Intervention}

Arthrocentesis was performed using an $18 \mathrm{G}$ needle by one of two experienced clinicians (TWON/NM) using a medial approach to the knee joint. Any synovial fluid obtained was forwarded for synovial fluid analysis. Using the same needle, the knee was then injected with $80 \mathrm{mg}$ methylprednisolone (without local anaesthetic). Any subject in whom the synovial fluid white cell count (WCC) was found to be greater than $1.5 \times 10^{9} / \mathrm{L}$ was subsequently withdrawn from the study because of a concern that they may not have OA.

\section{Follow-up}

We endeavoured to see all subjects within 14 days of injection. They completed the KOOS and VAS scores and had a repeat CE MRI. Response was assessed using the Outcome Measures in Rheumatology Clinical trials (OARSI-OMERACT) responder criteria using the KOOS pain scale to determine responder status. ${ }^{13}$ A responder was defined as either (i) a greater or equal to $20 \%$ change in KOOS pain score, with an absolute change of at least 3 units if the baseline score was 15 or less, and a global improvement in pain using a 5 category variable, or (ii) a greater or equal to a $50 \%$ change in the KOOS pain score with an absolute change of at least 3 units if the baseline score was 15 or less. Those who did not respond were not followed up. Those who responded were followed up by regular telephone calls during which the same KOOS survey questions were administered. Those whose pain recurred to within 20\% (of the baseline KOOS pain subscale score) were defined as having relapsed and were invited for a final MRI. Those whose pain levels did not return to this level at 6 months of follow-up were classified as having 'persistently responded' and had a final MRI scan scheduled at which point the study ended (see figure 1).

\section{Magnetic resonance imaging: acquisition and analysis}

Using a 3T Philips MRI, we obtained sagittal postcontrast T1W FS (TR $550 \mathrm{~ms}$, TE $20 \mathrm{~ms}$, FOV $14 \mathrm{~cm} \times 14 \mathrm{~cm}$, size $320 \times 320$ ) and sagittal precontrast 3D WATSc (TR $20 \mathrm{~ms}$, TE4.7 ms, FOV $15 \mathrm{~cm} \times 15 \mathrm{~cm}$, size $288 \times 288)$ scans in all subjects at baseline, within 2 weeks of follow-up and at a third, final visit, for patients whose symptoms recurred within 6 months or if they had not recurred at 6 months. An example of a postcontrast sagittal image from the study is shown in figure 2. Manual segmentation of the synovial tissue layer was performed on the sagittal postcontrast T1W FS image by a single observer. To optimise the ability to detect changes in synovial volume, segmentations were carried out paired though blinded to orderrepeated MRIs of a specific knee were segmented before moving to the next knee, with the visit order randomised by a separate member of the research team who took no part in the segmentation. Using computer image analysis, we excluded the cartilage within the segmented space by thresholding in the associated sagittal (3D WATSc: TR $20 \mathrm{~ms}$, TE $7.7 \mathrm{~ms}, F o V 16 \mathrm{~cm}$, $288 \times 288)$ scan. The rest of the segmented space was assumed to be a mixture of fluid and synovial tissue. We calculated the proportion of synovial tissue in every voxel using $\mathrm{P}=\left(\mathrm{I}-\mathrm{m}_{\mathrm{f}}\right) /$ $\left(m_{s}-m_{f}\right)$ truncated to $[0,1]$, where $I$ is the voxel intensity, and $m_{f}$ and $m_{s}$ are the means of the intensity distributions of fluid and STV, respectively. To evaluate repeatability of synovial volumes, the segmenter was asked to segment 10 knees randomly selected (without replacement) from those that had been previously segmented with new identification numbers that were assigned. In addition, a sample of 101 patients' images (262 images in total, across the three study visits) were evaluated semi-quantitatively 
Figure 1 Study flow diagram.

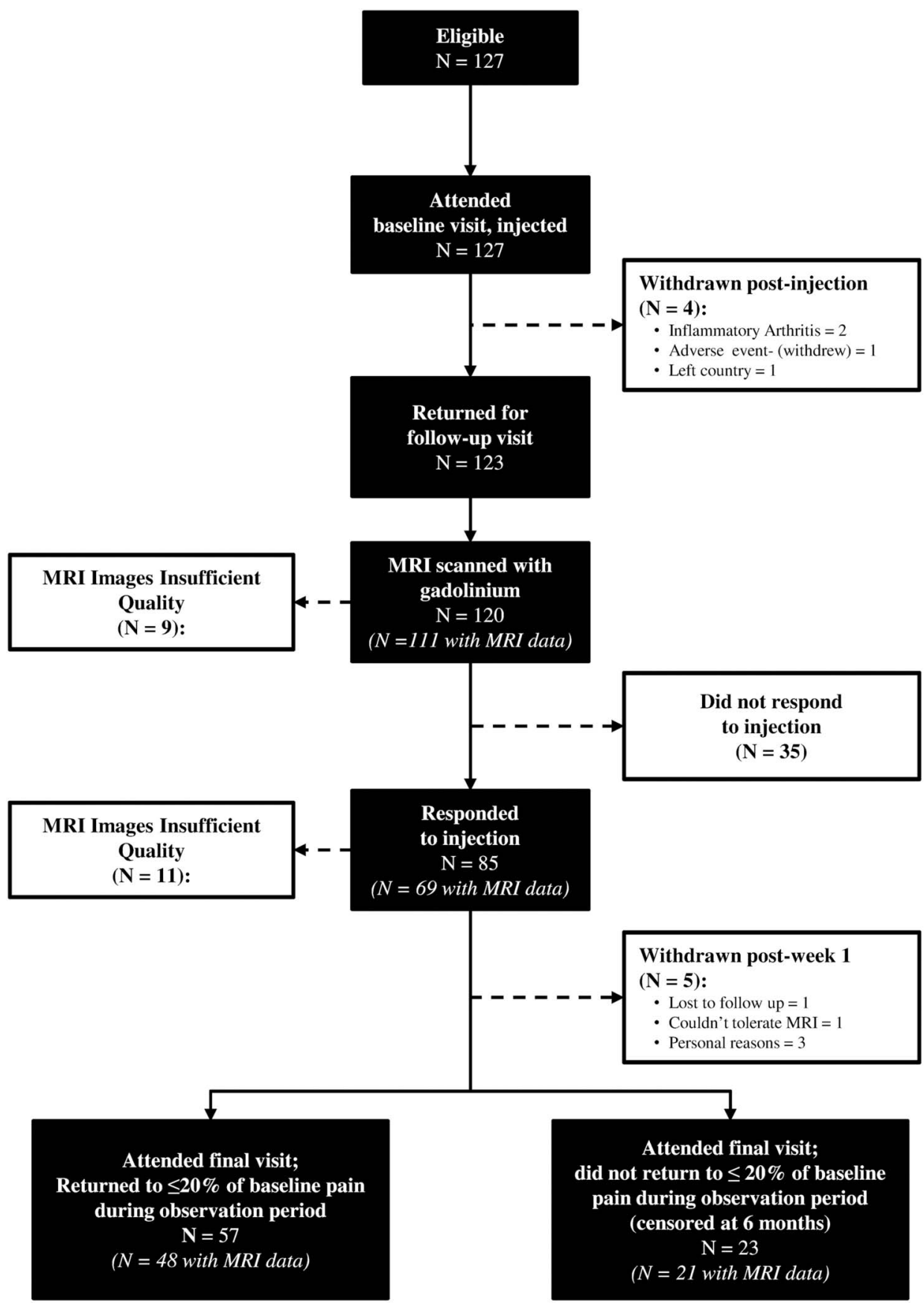

for the presence of synovitis by an experienced radiologist using an approach previously described, ${ }^{14}$ which comprised scoring each of 11 areas from $0-1-2$ in increasing order of severity, with a resulting total score up to 22 .

\section{Statistical analysis}

Subject characteristics and STVs were described with means and SDs for normally distributed variables and medians and interquartile ranges for variables with a skewed distribution. We assessed intraobserver repeatability of the manual segmentation process using intraclass correlation (ICC). The association between quantitative and semiquantitative approaches to assessing STV was evaluated using Spearman's correlation coefficient.

The primary outcome was to assess change in knee pain from baseline to the first follow-up visit. We calculated means and 95\% CIs for change in pain using the KOOS and $\mathrm{VAS}_{\mathrm{NA}}$, and STV between baseline and the postinjection visit. The means and CIs were calculated for those who were and those who were not OARSI responders separately. Following this, we looked at within-person change in symptoms and STV between the follow-up visit and final visit. To do this, we calculated means and 95\% CIs for within-person change for all patients who had responded to treatment and separately for patients whose symptoms had relapsed within the 6-month observation window and those whose symptoms had not relapsed during the 6 months of study (the 'persistent responders').

To evaluate whether there was an association between change in pain and change in synovitis across all three study visits, we used fixed-effects multiple linear panel regression (generalised multiple linear regression). The two models considered used either the KOOS pain score or $\mathrm{VAS}_{\mathrm{NA}}$ as the outcome variable, the STV and study visit (coded as dummy variables) as the predictor variables, and the subject ID as a panel variable. Formally written, the first model was therefore $y_{i t}=X_{i t 1} \beta+X_{i t 2} \beta+\alpha_{i}+u_{i t}$, 


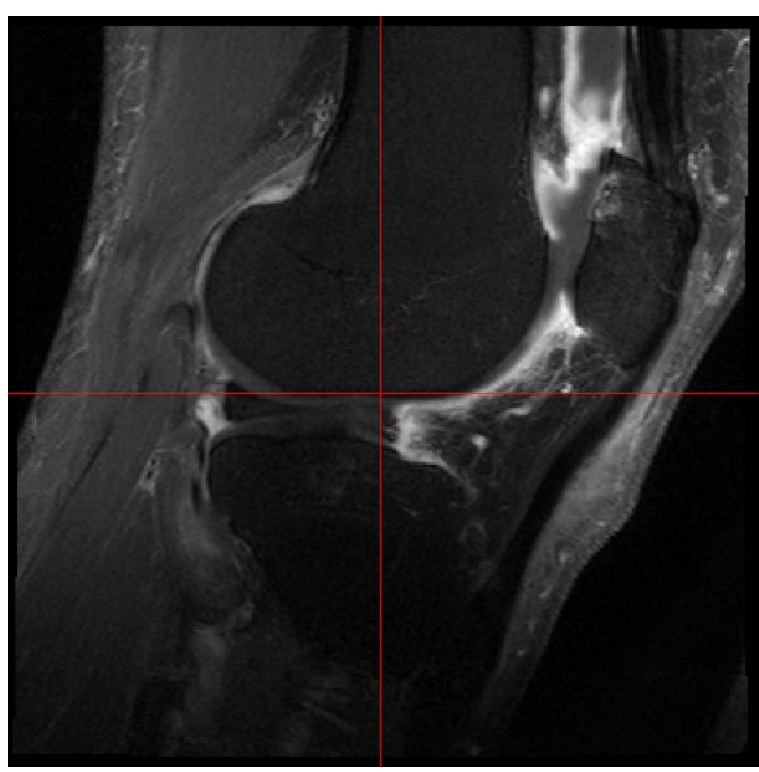

Figure 2 Gadolinium-enhanced MRI of study patient, showing synovial tissue and fluid differentiation.

where $y_{i t}=$ KOOS pain subscale score, and $X_{i t 1}=S T V$, $\mathrm{X}_{\mathrm{it} 2}=$ study visit (coded as dummy variables), $\alpha_{\mathrm{i}}=$ subject and $\mathrm{u}_{\mathrm{it}-}$ $=$ error. In the second model tested, $\mathrm{VAS}_{\mathrm{NA}}$ was the outcome variable (ie, $y_{i t}=V_{A A}$ ), but was otherwise identical to the first model. Statistical analysis was undertaken using Stata V.13.1. ${ }^{15}$

\section{RESULTS}

\section{Subjects}

A total of 127 subjects were recruited and administered a baseline steroid injection. Of these, two were excluded because their synovial fluid WCC was greater than $1.5 \times 10^{9} / \mathrm{L}$, one was withdrawn because of an adverse event (urinary tract infection), and

Table 1 Subject characteristics at baseline visit

\begin{tabular}{|c|c|}
\hline Variable & Statistic $(N=120)$ \\
\hline Age (years), mean (SD) & $62.3(10.3)$ \\
\hline Females, frequency (\%) & $62(51.7)$ \\
\hline $\begin{array}{l}\text { Number of days to follow-up appointment, median } \\
\text { (IQR) }\end{array}$ & $8.0(7.0$ to 13.5$)$ \\
\hline KOOS pain subscale score $(0-100)^{*}$, median (IQR) & 44.4 (36.1 to 55.6$)$ \\
\hline Pain on nominated activity VAS (0-10)t, median (IQR) & $7.0(5.5$ to 7.7$) \ddagger$ \\
\hline Pain in last week VAS (0-10)t, median (IQR) & 6.5 (4.7 to 7.8$) \ddagger$ \\
\hline $\begin{array}{l}\text { Maximal Kellgren-Lawrence grade in either } \\
\text { patellofemoral or tibiofemoral compartment }\end{array}$ & - \\
\hline Grade 2, frequency (\%) & $42(38.5) \S$ \\
\hline Grade 3, frequency (\%) & $60(55.1) \S$ \\
\hline Grade 4, frequency (\%) & $7(6.4) \S$ \\
\hline Synovial tissue volume $\left(\mathrm{mm}^{3}\right)$, median (IQR) & 8177 (5743 to 13 056) १ \\
\hline Synovial fluid volume $\left(\mathrm{mm}^{3}\right)$, median (IQR) & 8158 (5699 to 12457$)$ १ \\
\hline $\begin{array}{l}\text { Number of responders to injection, at follow-up visit, } \\
\text { frequency }(\%)\end{array}$ & $85(70.8)$ \\
\hline \multicolumn{2}{|c|}{$\begin{array}{l}\text { *KOOS pain subscale is scored from } 100 \text { (no pain) to } 0 \text { (extreme pain). } \\
\text { tVASs are scored from } 0 \text { (no pain) to } 10 \text { (pain as bad as you can imagine). } \\
\text { fFive patients and three patients neglected to complete their pain on nominated } \\
\text { activity VAS and pain in last week VAS, respectively. } \\
\text { SKellgren-Lawrence data is available only for } 115 \text { patients in total; the remaining } 12 \\
\text { were assessed for study eligibility via MRI or arthroscopy report. } \\
\text { INine patients' MRI images were of insufficient quality to allow volume calculation. } \\
\text { KOOS, Knee Injury and Osteoarthritis Outcome Score; VAS, Visual Analogue Scale. }\end{array}$} \\
\hline
\end{tabular}

a further subject was lost to follow-up. In total, 64 subjects (out of the 127 subjects injected) had fluid aspirated from their knee (50.4\%); the median volume was $4 \mathrm{~mL}$ (IQR 1.5 to $8.5 \mathrm{~mL}$; range $0.25-70 \mathrm{~mL}$ ). Of these, nine had evidence of crystals (calcium pyrophosphate (5), apatite (4)). Of the 109 subjects with radiographs the majority were either grade $2(38.5 \%)$ or grade $3(55.1 \%)$ with the rest grade $4(6.4 \%)$; see table 1 . Of those with tibiofemoral disease, the majority were medial (76.6\%) with only a small proportion (3.7\%) lateral. Just over two-thirds (64.9\%) had, in addition, significant patellafemoral involvement. Of the 123 who returned for their postinjection visit, three did not have CE MRI, because they experienced adverse events following the injection (see figure 1) and were therefore excluded from the analysis. The mean age of the 120 subjects in whom it was possible to assess STV at baseline and at the postinjection visit was 62.3 years (SD 10.3) and 62 (52\%) were women; see table 1 . Median KOOS pain score at baseline was 44.4 points (IQR 36.1-55.6), and median $\mathrm{VAS}_{\mathrm{NA}}$ was $7.0 \mathrm{~cm}$ (IQR 5.5-7.7). The median time between baseline and follow-up scan was 8 days (IQR 7-13.5).

\section{Assessment of STV}

Of the 120 subjects with preinjection and postinjection CE films, data on STV were available for 111 at baseline and follow-up. In nine subjects, no postsagittal image was taken or the quality of the image was considered to be poor, precluding comparison. The ICC for intraobserver reliability of the manual segmentation was excellent at 0.94 (95\% CI 0.81 to 0.98$)$. The semiquantitative and quantitative approaches to assessing STV were correlated $\left(r_{s}=0.70 ; 95 \%\right.$ CI 0.64 to 0.76$)$. The median STV at baseline was $8177 \mathrm{~mm}^{3}$ (IQR 5743-13 $056 \mathrm{~mm}^{3}$ ).

\section{Outcome following steroid injection}

Early outcome (baseline to postinjection visit)

Of the 120 who completed a postinjection CE MRI, 85 (71\%) were defined as responders (OARSI/OMERACT) and 35 as non-responders. Following injection, five subjects did not return for a final scan-this was for personal reasons (3), loss to follow-up (1) and inability to tolerate the scan (1). Table 2 summarises the changes in symptoms and also the STV between baseline and the postinjection visit. Following injection, knee pain significantly improved on both the KOOS (+23.9 points; $95 \%$ CI 20.1 to $27.8 ; \mathrm{p}<0.001)$ and $\mathrm{VAS}_{\mathrm{NA}}(-3.2 \mathrm{~cm} ; 95 \% \mathrm{CI}$ -3.8 to $-2.7 ; \mathrm{p}<0.001)$. There was a reduction also in mean synovitis volume $\left(-1071 \mathrm{~mm}^{3} ; 95 \%\right.$ CI -1839 to -303 ; $\mathrm{p}=0.01)$. Those defined as responders had a greater improvement in pain than those who did not respond (between group difference in $\mathrm{KOOS}=33.1$ points; 95\% CI 27.2 to 39.1 ; $\mathrm{p}<0.001 .8$ and $\mathrm{VAS}_{\mathrm{NA}}=-3.5 \mathrm{~cm} ; 95 \% \mathrm{CI}-4.5$ to -2.6 ; $\mathrm{p}<0.001)$. There was no difference in the change in pain or STV in those who had evidence of crystals in their synovial fluid at baseline and those with fluid, but without crystals (tests for interaction effects for change-by-crystal presence in linear regressions assessing change in the KOOS pain score, $\mathrm{VAS}_{\mathrm{NA}}$ and STV were $0.72,0.89$ and 0.22 , respectively). There was no difference in $\mathrm{K} / \mathrm{L}$ grade between responders and non-responders $\left(\chi^{2}(2, N=111)=0.02 ; p=0.99\right)$.

\section{Late outcome (postinjection to final scan)}

Of the 85 subjects who responded, five did not return for a third MRI scan. Of the 80 subjects in whom MRI was performed, 57 (71\%) had a relapse within 6 months of their injection and 23 (29\%) did not (the 'persistent responders'). Table 3A, B summarises the changes in symptoms and also STV 
Table 2 Synovial tissue volume and pain: baseline and follow-up visits

\begin{tabular}{|c|c|c|c|c|c|c|}
\hline Variable & $\begin{array}{l}\text { Baseline visit } \\
\text { Mean (SD) }\end{array}$ & $\begin{array}{l}\text { Follow-up visit } \\
\text { Mean (SD) }\end{array}$ & $\begin{array}{l}\text { Difference* } \\
\text { Mean }(95 \% \mathrm{Cl})\end{array}$ & $\mathrm{p}$ Value & $\begin{array}{l}\text { Difference; non-responderst } \\
\text { Mean }(95 \% \mathrm{Cl})\end{array}$ & $\begin{array}{l}\text { Difference; responders } t \\
\text { Mean }(95 \% \mathrm{Cl})\end{array}$ \\
\hline Synovitis volume $\left(\mathrm{mm}^{3}\right)$ & $9935(6470)$ & $8864(7802)$ & $-1071(-1839$ to -303$)$ & 0.01 & $-159(-1553$ to 1235$)$ & $-1474(-2401$ to -548$)$ \\
\hline Synovial fluid volume $\left(\mathrm{mm}^{3}\right)$ & $9872(6852)$ & 9019 (7449) & $-853(-1615$ to -90$)$ & 0.03 & $-416(-1797$ to 964$)$ & $-1045(-1962$ to -128$)$ \\
\hline KOOS pain subscale scoreł $(0-100)$ & $45.6(14.6)$ & $69.5(19.8)$ & 23.9 (20.1 to 27.8 ) & $<0.001$ & 0.5 (-4.6 to 5.5$)$ & 33.6 (30.4 to 36.8$)$ \\
\hline Pain on Nominated Activity VAS (0-10) & $6.53(1.8)$ & $3.30(2.7)$ & $-3.23(-3.8$ to -2.7$)$ & $<0.001$ & $-0.7(-1.5$ to 0.2$)$ & $-4.2(-4.8$ to -3.7$)$ \\
\hline
\end{tabular}

between the postinjection and relapse visit. Among those whose pain relapsed (57), there was a significant worsening of their pain including KOOS (-27.7 points; $95 \%$ CI -32.0 to -23.4 ; $\mathrm{p}<0.001)$ and $\operatorname{VAS}_{\mathrm{NA}}(+4.4 \mathrm{~cm} ; 95 \%$ CI 3.7 to $5.1 ; \mathrm{p}<0.001)$. In addition, there was a significant increase in STV (withinperson change $=+1220 \mathrm{~mm}^{3} ; 95 \%$ CI 25 to $2414 ; \mathrm{p}=0.05$ ). Among those whose pain did not relapse (23), there was a small within-person worsening in their KOOS pain score $(-10.6$; $95 \% \mathrm{CI}-17.3$ to $-3.8 ; \mathrm{p}<0.001)$ and also $\mathrm{VAS}_{\mathrm{NA}}(1.3 ; 95 \%$ CI 0.2 to $2.4 ; \mathrm{p}=0.02)$, though there was no significant change in STV $\left(-202 \mathrm{~mm}^{3} ; 95 \% \mathrm{CI}-2008\right.$ to $\left.1604 ; \mathrm{p}=0.83\right)$.

\section{Correlation between symptoms and structure}

Using generalised linear modelling and adjusting for the effect of person and time (all three study visits), there was a significant association between synovitis volume and pain score (for each of KOOS and $\mathrm{VAS}_{\mathrm{NA}}, \mathrm{p}<0.0001$ ), see figure 3 and table 4 . The increase in variance explained by the inclusion of STV in the models was, however, relatively small (change in $\mathrm{R}^{2}$ is 0.02 for the KOOS model and 0.04 for the $\mathrm{VAS}_{\mathrm{NA}}$ model). Limiting the analyses to all initial responders (later persistent responders and those who failed) and examining pain change after initial response to later evaluation, there was a significant association between change in the level of synovitis and change in level of pain (for KOOS, $p=0.01$; for $\operatorname{VAS}_{\mathrm{NA}} \mathrm{p}=0.04$ ). There was no evidence of interaction between group status (persistent responder/failed) on this association.

\section{DISCUSSION}

In this open label study in men and women with symptomatic knee OA, there was a significant improvement in knee pain and a reduction in STV following intra-articular steroid injection, with a significant correlation between change in pain and change in STV. Among those whose pain recurred within 6 months, both pain and STV increased. A significant correlation was observed between increasing pain and increasing STV, when patient and time effects were adjusted for. Taken together, these data suggest that STV is a good candidate to be a treatment target in knee OA.

In this study, we used contrast enhancement to quantify synovial volume. Non-contrast-enhanced scans are unable to differentiate synovial tissue from synovial fluid. Consequently, the presence of synovial fluid resulted in misclassification. Our method involved manual segmentation of the outer layer of enhanced synovium and, to minimise errors of segmentation, used image analysis to demarcate the area of synovium from the surrounding lower-intensity areas including synovial fluid and cartilage. Assessment of STV is subject to errors of precision including segmentation-though formal testing suggested excellent intraobserver repeatability. Furthermore, there was good correlation between STV assessed quantitatively and semiquantitatively. Indeed, where STV was higher there was some evidence that the assessment of STV may have been more sensitive than in the semi-quantitative approach, which is constrained to a maximum score of 2 at any one of the 11 target evaluation sites (data not shown). In our study, there was no significant correlation between the aspirated synovial fluid volume and the decrease in STV between baseline and the immediate postinjection visit (Spearman's correlation coefficient $\mathrm{R}=-0.17$ ) suggesting that STV did not incorporate free fluid in the joint. Our data suggest that STV assessed quantitatively is a reliable and valid method of assessment of synovitis in knee OA.

Table 3 Synovial tissue volume and pain: baseline, follow-up and final visits, split by response type

\begin{tabular}{|c|c|c|c|c|c|}
\hline Variable & $\begin{array}{l}\text { Baseline, } \\
\text { mean (SD) }\end{array}$ & $\begin{array}{l}\text { Follow-up, } \\
\text { mean (SD) }\end{array}$ & $\begin{array}{l}\text { Final Visit, } \\
\text { mean (SD) }\end{array}$ & $\begin{array}{l}\text { Difference; Baseline to } \\
\text { follow-up Visit } \\
\text { Mean (95\% Cl) }\end{array}$ & $\begin{array}{l}\text { Difference; Follow-up } \\
\text { to final Visit } \\
\text { Mean }(95 \% \mathrm{Cl})\end{array}$ \\
\hline \multicolumn{6}{|c|}{ (A) Initial responders, whose pain recurred within 6 months ('Relapsers') } \\
\hline Synovitis volume $\left(\mathrm{mm}^{3}\right)$ & $10319(5419)$ & 8609 (5517) & $9829(5048)$ & $-1710(-2904$ to -515$)$ & 1220 (25 to 2414$)$ \\
\hline Synovial fluid volume $\left(\mathrm{mm}^{3}\right)$ & $10023(5408)$ & $8690(5514)$ & $9860(5152)$ & $-1334(-2555$ to -113$)$ & 1170 (-51 to 2391$)$ \\
\hline KOOS pain subscale score $(0-100)$ & $45.1(13.6)$ & $75.57(14.7)$ & $47.89(17.1)$ & 30.42 (26.1 to 34.7$)$ & $-27.7(-32.0$ to -23.4$)$ \\
\hline Pain on nominated activity VAS (0-10) & $6.5(1.5)$ & $1.99(1.8)$ & $6.41(2.3)$ & $-4.5(-5.2$ to -3.8$)$ & 4.4 (3.7 to 5.1$)$ \\
\hline \multicolumn{6}{|c|}{ (B) Initial responders, whose pain did not recur within 6 months ('Persistent responders') } \\
\hline Synovitis volume $\left(\mathrm{mm}^{3}\right)$ & 8627 (5308) & $7782(4436)$ & 7580 (5401) & $-845(-2651$ to 961$)$ & $-202(-2008$ to 1604$)$ \\
\hline Synovial fluid volume $\left(\mathrm{mm}^{3}\right)$ & $8676(5602)$ & $8322(5407)$ & $7663(5323)$ & $-355(-2201$ to 1491$)$ & $-658(-2504$ to 1188$)$ \\
\hline KOOS pain subscale score $(0-100)$ & $42.9(15.9)$ & $83.9(12.5)$ & $73.3(17.7)$ & $41.0(34.3$ to 47.8$)$ & $-10.6(-17.3$ to -3.9$)$ \\
\hline Pain on nominated activity VAS (0-10) & $6.5(1.6)$ & $2.4(2.2)$ & $3.6(2.5)$ & $-4.1(-5.2$ to -3.0$)$ & $1.3(0.2$ to 2.4$)$ \\
\hline
\end{tabular}

KOOS, Knee Injury and Osteoarthritis Outcome Score; VAS, Visual Analogue Scale. 
Figure 3 Relationship between Knee Injury and Osteoarthritis Outcome Score (KOOS) pain subscale score and synovial tissue volume (STV).

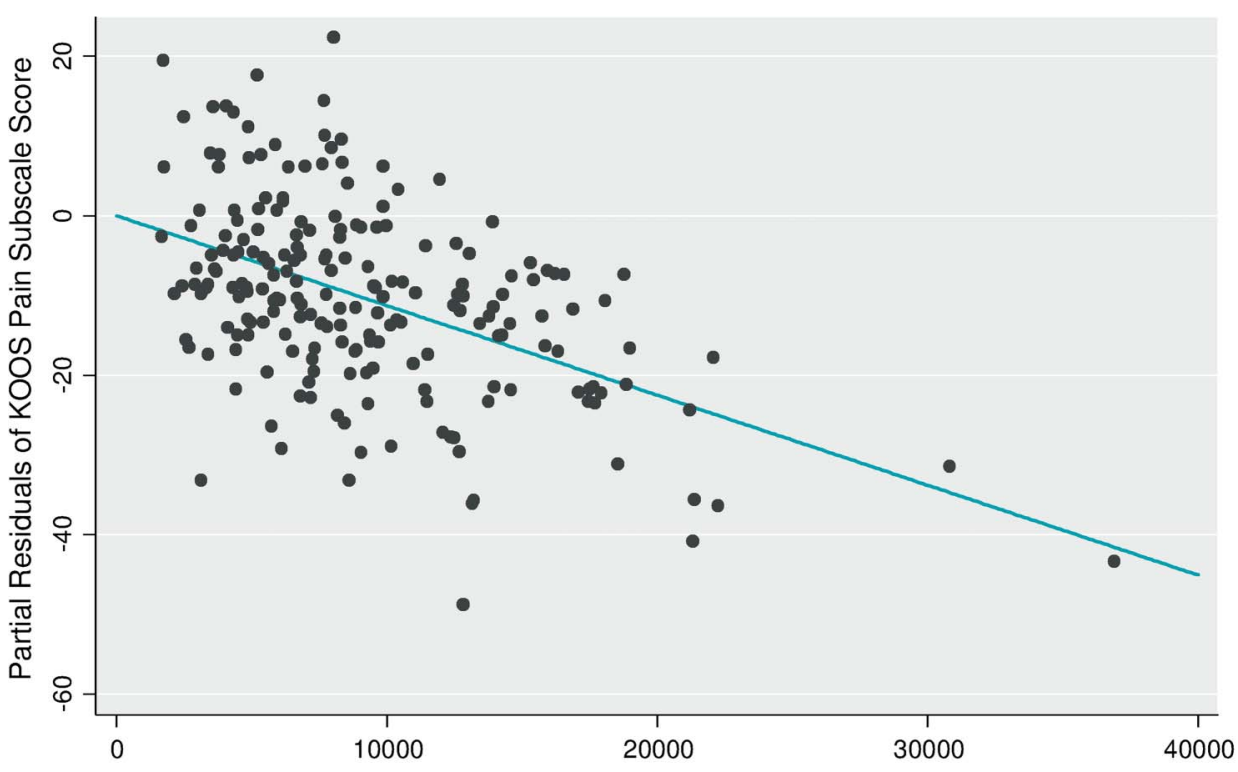

Synovitis Volume $\left(\mathrm{mm}^{3}\right)$

$$
\text { — Synovits Volume Effect (beta) }
$$

Figure above is a partial-residual plot from a fixed-effects panel model with KOOS pain subscale score as the outcome variable, STV, and study visit (coded as dummy variables) as the predictor variables, and subject ID as the fixed-effects estimator. Formally written, this model is $y_{i t}=X_{i t 1} \beta+X_{i t 2} \beta+\alpha_{i}+u_{i t}$, where $y_{i t}=$ KOOS pain subscale score, $X_{i t 1}=$ synovial tissue volume (STV), $X_{i t 2}=$ study visit (coded as dummy variables), $\alpha_{i}=$ subject, and $u_{i t}=$ error.

The line depicts the partial coefficient (b) for STV, shown in Table 4
In our study, among those with serial MRI data at baseline and follow-up, the overall response rate for steroid injection was $71 \%$. Of the 85 subjects who initially responded to injection, just over $25 \%$ were, based on OARSI-OMERACT criteria, persistent responders at 6 months, though with a trend towards increasing pain among this group. We used a higher dose of steroids ( $80 \mathrm{mg}$ depomedrone) than others which may in part explain a longer-term response. ${ }^{10}$ We found a prolonged effect of steroids in some patients, and this was accompanied by a continuing suppression of synovitis, suggesting that a minority of patients with knee OA might be treated successfully with intermittently repeated steroid injections. This finding has important clinical implications and needs to be corroborated in other samples. Since we did not include a placebo control group in this study, we cannot rule out that these patients may have had inherently episodic disease, perhaps induced by crystals.

Steroids are widely used in the management of knee OA and it is assumed that their analgesic effect is related to their antiinflammatory properties. Inflammation has long been recognised as a feature of knee OA, though recent studies using MRI scanning suggest that it may be more common than previously thought with up to $90 \%$ of subjects having evidence of increased STV on MRI scanning. ${ }^{2}$ Inflammation is likely secondary to release of cartilage degradation products into the joint and consequent triggering of the inflammatory cascade within the synovial lining layer manifesting as synovitis.

What about the impact of non-intra-articular targeted therapy on synovial tissue? In a recent open label study, 36 patients with symptomatic hand OA were given $120 \mathrm{mg}$ of intramuscular methylprednisolone. There was a significant improvement in symptoms after 4 weeks and a non-significant trend for OARSI responders to have higher levels of baseline synovitis as assessed using ultrasound. ${ }^{16}$ In a more recent Egyptian study, 144 patients with symptomatic knee OA were randomised to receive either methotrexate of placebo. After 28 weeks there was a clinically relevant reduction in knee symptoms and also synovitis, assessed using ultrasound, in the methotrexate group compared with placebo. ${ }^{17}$ No attempt though was made to correlate the change in synovitis and pain in this study; however, these data support the view that synovitis may be a structural target in knee OA.

To our knowledge, our study is the largest of intra-articular therapy in knee OA, and the only one in which CEMRI was used to assess outcome and at multiple time points. There are a number of limitations to be considered. This was an open label study with subjects aware of the intervention; consequently, it is not possible to isolate the effect of the intervention on symptoms or structural outcomes as would be possible in a placebocontrolled trial. While some of the pain reduction could be due to regression to the mean or to a placebo response, it is unlikely that any of the observed structure-symptom relationships could be explained on the basis of a 'placebo' or contextual effect of the intervention. We note that the overall response was similar to that observed in other studies. The injections in this study were administered without imaging guidance, and it is possible that inaccuracy in placement may have contributed to a reduction in effect. While this may have affected the overall response rate, it seems unlikely that any inaccuracy in localisation of the injection to within the joint would have impacted the structural-symptom relationship that was a focus of this study.

In this study, we have shown that STV shrinks following antiinflammatory therapy, that in most patients it rebounds after an interval period and changes in pain are linked with changes in synovitis. The correlation between change in pain and change in STV was small, supporting the view that other structural and non-structural factors play a substantial role in the occurrence of pain in knee OA. Further, the weak, although significant, 
Table 4 Relationship between STV and pain scores

\begin{tabular}{|c|c|c|c|c|}
\hline \multirow[b]{2}{*}{ Model outcome } & \multicolumn{4}{|c|}{ Effect of change in STV on outcome $\left(\mathrm{mm}^{3}\right)$} \\
\hline & $b^{*}(95 \% \mathrm{Cl})$ & p Value & $\boldsymbol{\beta}$ & Change in $R^{2}$ overallt \\
\hline KOOS pain subscale score $(0-100)$, mean (SD) & $-1.13(-1.87$ to -0.39$)$ & 0.003 & -0.28 & 0.02 \\
\hline Pain on nominated activity VAS (0-10), mean (SD) & $0.17(0.05$ to 0.29$)$ & 0.006 & 0.32 & 0.04 \\
\hline
\end{tabular}

relationship of synovial volume and pain change suggests that it may be a viable structural target in trials in OA. It may be challenging to show causal associations between the two parameters without large numbers in these trials. Even so, our data suggest that synovitis can be considered a candidate treatment target for anti-inflammatory therapies in knee OA which focus on their analgesic effect.

In conclusion, synovitis shrinks following steroid therapy in knee $\mathrm{OA}$ and its fluctuation correlates with the severity of knee pain. Synovitis should be considered a structural target for treatment in symptomatic knee OA.

\author{
Author affiliations \\ ${ }^{1}$ Faculty of Medical and Human Sciences, Arthritis Research UK Centre for \\ Epidemiology, Institute of Inflammation and Repair, Manchester Academic Health \\ Science Centre, University of Manchester, Manchester, UK \\ ${ }^{2}$ NIHR Manchester Musculoskeletal Biomedical Research Unit, Central Manchester \\ NHS Foundation Trust, Manchester Academic Health Science Centre, Manchester, UK \\ ${ }^{3}$ Department of Rheumatology, Salford Royal NHS Foundation Trust, Salford, UK \\ ${ }^{4}$ Centre of Imaging Sciences, Institute of Population Health, University of \\ Manchester, Manchester, UK \\ ${ }^{5}$ UK Warwick Medical School, The University of Warwick, Coventry, UK \\ ${ }^{6}$ Clinical Epidemiology Unit, Boston University School of Medicine, Boston, \\ Massachusetts, USA
}

Twitter Follow Research in Osteoarthritis Manchester (ROAM) at @ROAM_Research

Acknowledgements The authors appreciate the expert assistance of Helen Williams, Laura Heathers, Laura Forsythe, Rosie Perry, and the assistance of the rest of the ROAM team. The authors are thankful for the generous contributions of time and energy of study subjects and appreciate the expertise and assistance of iMorphics staff and especially Mike Bowes, PhD. The authors would like to acknowledge the equipment and facilities provided by the Salford Royal NHS Foundation Trust.

Contributors DTF initially proposed the study. TWON and DTF wrote the protocol. TWON, DTF and MJP wrote the manuscript. TWON, NM, RH, CEH and EJM collected the data. TWON, MJP, RH, ADG, TFC, CEH analysed the data. TWON, MJP, NM, RH, ADG, TFC, CEH, DTF and EJM reviewed drafts of the paper.

Funding This review was funded by Arthritis Research UK grant 20380, and special strategic award grant 18676. This report includes independent research supported by (or funded by) the National Institute for Health Research Biomedical Research Unit Funding Scheme. The views expressed in this publication are those of the author(s) and not necessarily those of the NHS, the National Institute for Health Research or the Department of Health. The Research in Osteoarthritis Manchester (ROAM) group is supported by the Manchester Academic Health Sciences Centre (MAHSC). DTF is supported by NIH AR4778. NM is supported by an NIHR Allied Health Professional Clinical Doctoral Fellowship.

Competing interests DTF reported that he serves as a consultant for Zimmer Knee Creations.

Ethics approval This study was approved by Leicestershire Multicentre Research Ethics Committee on 27 January 2010 (ref: 09/H0402/107).

Provenance and peer review Not commissioned; externally peer reviewed.

Data sharing statement The corresponding author (TWON) had full access to all the data in the study and takes responsibility for the integrity of the data, and the accuracy of the data analysis.
Open Access This is an Open Access article distributed in accordance with the terms of the Creative Commons Attribution (CC BY 4.0) license, which permits others to distribute, remix, adapt and build upon this work, for commercial use, provided the original work is properly cited. See: http://creativecommons.org/ licenses/by/4.0/

\section{REFERENCES}

1 Ayral X, Pickering EH, Woodworth TG, et al. Synovitis: a potential predictive factor of structural progression of medial tibiofemoral knee osteoarthritis-results of a 1 year longitudinal arthroscopic study in 422 patients. Osteoarthritis Cartilage 2005;13:361-7.

2 Roemer FW, Javaid KM, Guermazi A, et al. Anatomical distribution of synovitis in knee osteoarthritis and its association with joint effusion assessed on non-enhanced and contrast-enhanced MRI. Osteoarthritis Cartilage 2010;18:1269-74.

3 Loeuille D, Chary-Valckenaere I, Champigneulle J, et al. Macroscopic and microscopic features of synovial membrane inflammation in the osteoarthritic knee: correlating magnetic resonance imaging findings with disease severity. Arthritis Rheum 2005;52:3492-501.

4 Hill CL, Gale DG, Chaisson CE, et al. Knee effusions, popliteal cysts, and synovial thickening: association with knee pain in osteoarthritis. J Rheumatol 2001;28:1330-7.

5 Hill CL, Hunter DJ, Niu J, et al. Synovitis detected on magnetic resonance imaging and its relation to pain and cartilage loss in knee osteoarthritis. Ann Rheum Dis 2007;66:1599-603.

6 Zhang Y, Nevitt M, Niu J, et al. Fluctuation of knee pain and changes in bone marrow lesions, effusions, and synoivitis on magnetic resonance imaging. Arthritis Rheum 2011:63:691-9.

7 Roemer FW, Guermazi A, Zhang Y, et al. Hoffa's Fat Pad: evaluation on unenhanced MR images as a measure of patellofemoral synovitis in osteoarthritis. Am J Roentgenol 2009;192:1696-700.

8 Loeuille D, Sauliere N, Champigneulle J, et al. Comparing non-enhanced and enhanced sequences in the assessment of effusion and synovitis in knee OA: associations with clinical, macroscopic and microscopic features. Osteoarthritis Cartilage 2011;19:1433-9.

9 Baker KR, Grainger AJ, Niu JB. Synovitis is associated with knee pain in those without X-ray knee OA. Arthritis Rheum 2007;56(suppl):S123.

10 Arroll B, Goodyear-Smith F. Corticosteroid injections for osteoarthritis of the knee: meta-analysis. BMJ 2004;328:869.

11 Ostergaard M, Stoltenberg M, Gideon $\mathrm{P}$, et al. Changes in synovial membrane and joint effusion volumes after intraarticular methylprednisolone. Quantitative assessment of inflammatory and destructive changes in arthritis by MRI. J Rheumatol 1996;23:1151-61.

12 Roos EM, Roos HP, Lohmander LS, et al. Knee Injury and Osteoarthritis Outcome Score (KOOS) - development of a self-administered outcome measure. J Orthop Sports Phys Ther 1998;28:88-96.

13 Pham T, van der Heijde D, Altman RD, et al. OMERACT-OARSI initiative: Osteoarthritis Research Society International set of responder criteria for osteoarthritis clinical trials revisited. Osteoarthritis Cartilage 2004;12: 389-99.

14 Guermazi A, Roemer FW, Hayashi D, et al. Assessment of synovitis with contrast-enhanced MRI using a whole-joint semiquantitative scoring system in people with, or at high risk of, knee osteoarthritis: the MOST study. Ann Rheum Dis 2011;70:5 805-11.

15 StataCorp. Stata Statistical Software: Release 13. College Station, TX, USA: StataCorp LP., 2013.

16 Keen HI, Wakefield RJ, Hensor EM, et al. Response of symptoms and synovitis to intra-muscular methylprednisolone in osteoarthritis of the hand: an ultrasonographic study. Rheumatology 2010;49:1093-100.

17 Abou-Raya A, Abou-Raya S, Kadrawe T. Methotrexate in the treatment of symptomatic knee osteoarthritis: randomised placebo-controlled trial. Ann Rheum Dis 2014. Published Online First: 27 Mar 2014 doi:10.1136/annrheumdis-2013-204856. 